\title{
A Study of Service in Restaurant by using Queuing Model
}

\author{
G D Mishra ${ }^{1}$, Vijiya Singh Chauhan², Nikita Chandra ${ }^{3}$ \\ ${ }^{1}$ Professor, Department of Applied Sciences Amity University, Noida \\ ${ }^{2}$ Research Scholar, Department of Mathematics, Bhagwant University, Ajmer \\ ${ }^{3}$ Department of Applied Sciences, Amity University, Noida
}

Keywords: Queue; Little's Theorem; Restaurant; Waiting lines

\begin{abstract}
The restaurants want to avoid losing their customers due to a long wait on This shows a need of a numerical model for the restaurant management to underst id the sh tion better. This paper aims to show that queuing theory satisfies the model when tesco rith a real ase scenario. We obtained the data from a restaurant. We then derive the arr rat rervice rate, utilization rate, waiting time in queue and the probability of potential aston ars to $\mathrm{lk}_{\mathrm{k}}$ based on the data using Little's Theorem and M/M/1 queuing model. con ate paper by discussing the benefits of performing queuing analysis to a busy resta

\section{Introduction}

There are several factors for a restaurant to attract the castomers. The $\chi$ important factors are taste, cleanliness, the restaurant layout and settings. The factors, when panaged carefully, will be able to attract plenty of customers. However, then is also an/ther factor that needs to be considered especially when the restaurant has already s eeded in ttracting customers. This factor is the customers queuing time.

The queuing theory is the study of queue o lines. Some of the analysis that can be derived using queuing theory include the expec d arme time in the que the average time in the system, the expected queue length the exp cted number of customers served at one time, the probability of balking custor ers, well the probability of the system to be in certain states, such as empty or full.

The waiting lines are a gomol gnt restaurants especially during lunch and dinner time. Hence, queuing th $\mathrm{V}$ is suita to be applied in a restaurant setting since it has an associated queue or wai hg where culomers who cannot be served immediately have to queue (wait) for service. $M$ resea rs have previously used queuing theory to model the restaurant operation [2], red ce cycle time in busy fast food restaurant [3], as well as to increase throughput and efficiency

In this chapter w e queu $g$ theory to study the waiting lines in a restaurant. The restaurant provides 2 les on eore There 8 to 9 waiters working at any one time. On a daily basis, it sery ove 400 cus mers during weekdays and over 1000 customers during weekends. This paper s.

In 1908, enhagen Telephone Company requested Agner K. Erlang to work on the holding times in a phone switch. He identified that the number of telephone conversations and telephone hokaing time fit into Poisson distribution and exponentially distributed. This was the beginning of the study of queuing theory. In this section, we will discuss two common concepts in queuing theory.

Little's theorem [7] describes the relationship between throughput rate (i.e. arrival and service rate), cycle time and work in process (i.e. number of customers/jobs in the system). This relationship has been shown to be valid for a wide class of queuing models. The theorem states that the expected number of customers $(\mathrm{N})$ for a system in steady state can be determined using the following equation:

$$
\mathrm{L}=\lambda \mathrm{T}
$$


Where, $\lambda$ is the average customer arrival rate and $\mathrm{T}$ is the average service time for a customer. Consider the example of a restaurant where the customer's arrival rate $(\lambda)$ doubles but the customers still spend the same amount of time in the restaurant $(T)$. These facts will double the number of customers in the restaurant $(L)$. By the same logic, if the customer arrival rate $(\lambda)$ remains the same but the customers service time doubles this will also double the total number of customers in the restaurant. This indicates that in order to control the three variables, managerial decisions are only required for any two of the three variables.

Three fundamental relationships can be derived from Little's theorem [6]:

- $\quad L$ increases if $\lambda$ or $T$ increases

- $\quad \lambda$ increases if $L$ increases or $T$ decreases

- $\quad T$ increases if $L$ increases or $\lambda$ decreases

Rust [8] said that the Little's theorem can be useful in quantifying the maxi sum ach able operational improvements and also to estimate the performance change w/ the syste is modified.

The data were obtained from a restaurant through interview with the resta ant manag data collections through observations at the restaurant. The daily number of visitors was obtained from the restaurant itself. The tau has b h recording the data as part of its end of day routine. We also interviewed t restauran an ger to find out about the capacity of the restaurant, the number of waiters, as el the num of chefs in the restaurant. Based on the interview with the restaurant mere w ncluded that the queuing model that best illustrate the operation of a restaurant is

This means that the arrival and service time are expo entially distributed (Poisson process). The restaurant system consists of only one server. In our c ervation th restaurant has several waiters but in the actual waiting queue, they only have one chen verve all of the customers.

For the analysis of a restaurant $\mathrm{M} / \mathrm{M} / 1$ queuin model, tho ving variables will be investigated [6]:

-

\section{$\lambda:$ The mean customers arrival rate}

$\mu$ : The mean service rate $\rho: \lambda / \mu:$ utilization facto Probability of zero cy to.
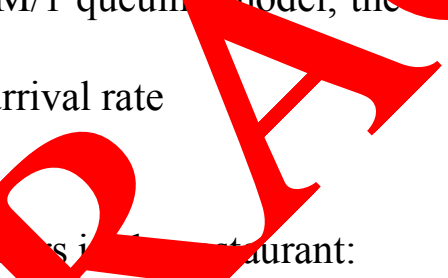

- $\mathrm{P}_{\mathrm{n}}$ : The proba of having, astomers in the restaurant.

L: aver ge nu ber of cus,omers dining in the restaurant.

$$
\mathrm{L}=\frac{\rho}{(1-\rho)}=\frac{\lambda}{(\mu-\lambda)}
$$

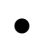

average number in the queue.

$$
\gamma \mathrm{Lq}=\mathrm{L} \times \rho=\frac{\rho^{2}}{(1-\rho)}=\frac{\rho \lambda}{(\mu-\lambda)}
$$

- W: average time spent in the restaurant, including the waiting time.

$$
\mathrm{W}=\frac{L}{\lambda}=\frac{1}{(\mu-\lambda)}
$$

- $\quad \mathrm{Wq}$ : average waiting time in the queue.

$$
\mathrm{Wq}=\frac{L_{q}}{\lambda}=\frac{\rho}{(\mu-\lambda)}
$$




\section{Data}

\begin{tabular}{|c|c|c|c|c|c|c|c|}
\hline & Mon & Tues & Wed & Thu & Fri & Sat & Sun \\
\hline $1^{\text {st }}$ Week & 470 & 427 & 429 & 492 & 663 & 973 & 1092 \\
\hline $2^{\text {nd }}$ Week & 456 & 445 & 536 & 489 & 597 & 1115 & 1066 \\
\hline $3^{\text {rd }}$ Week & 421 & 541 & 577 & 679 & 918 & 1319 & 1212 \\
\hline $4^{\text {th }}$ Week & 494 & 559 & 581 & 613 & 697 & 1188 & 1113 \\
\hline
\end{tabular}

\section{Calculation}

We conducted the research at dinner time. There are on average 400 the restaurant in 3 hours time of dinner time. From this we can derive the ar aval rate as

$$
\lambda=\frac{400}{1800}=2.22 \text { customers } / \text { minutes }(\mathrm{cpm}
$$

We also found out from observation and discussion with man that each spends 55 minutes on average in the restaurant $(W)$, the queue length aro 36 people $(L q)$ on average and the waiting time is around 15 minutes.

It can be shown using (7) that the observed actual $y$ diting time does, rot differ by much when compared to the theoretical waiting time as shown belo

$$
\mathrm{Wq}=\frac{36 \text { customers }}{2.22 c p} 16.22 \text { mares }
$$

Next, we will calculate the average num of peo, e in the restaurant using (1).

$$
\mathrm{L}=2.2 \mathrm{cpm} 55 \mathrm{minu} \mathrm{s}=122.1 \text { customers }
$$

Having calculated the avera on the restaurant, we can also derive the utilization rate and the sery rate using

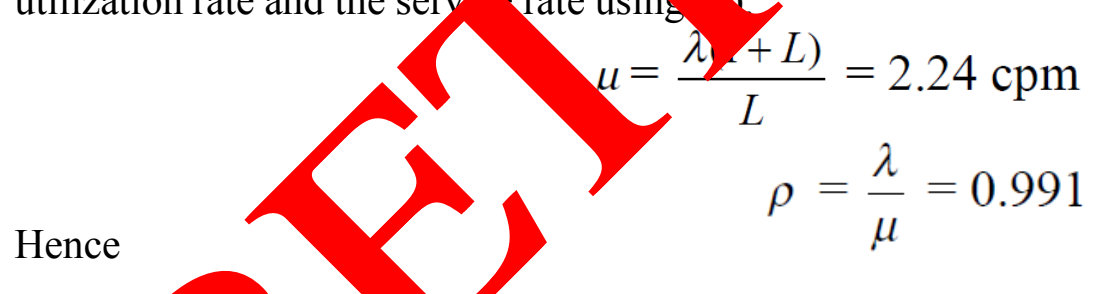

With the probability of zero customers in the restau is very small as can be derived using (2).

$$
\mathrm{P}_{0}=1-\rho=0.019
$$

The generic formula that can be used to calculate the probability of having $n$ customers in the restaurant is as follows:

$$
P_{n}=(1-0.991)(0.991)^{n}=(0.019)(0.991)^{n}
$$

We assume that potential customers will start to balk when they see more than 10 people are already queuing for the restaurant. We also assume that the maximum queue length that a potential customer can tolerate is 40 people. As the capacity of the restaurant when fully occupied is 120 people, we can calculate the probability of 10 people in the queue as the probability when there are 130 people in the system (i.e. 120 in the restaurant and 10 or more queuing) as follows: 
Probability of customers going away $=\mathrm{P}$ (more than 15 people in the queue)

$=\mathrm{P}$ (more than 130 people in the restaurant)

$$
\mathrm{P}_{131 \text { to } 160}=\sum_{n=131}^{160} P_{n}=0.1534=15.34 \%
$$

\section{Evaluation}

The utilization is directly proportional with the mean number of customers. It means that the mean number of customers will increase as the utilization increases.

- The utilization rate at the restaurant is very high at 0.991 . This, however, is only zation rate during lunch and dinner time on Saturdays and Sundays. On weekday, the tilization te is almost half of it. This is because the number of visitors on weekdays is only of the nu ber of visitors on weekends. In addition, the number of waiters remains the s? rega it is peak hours or off-peak hours.

- In case the customers waiting time is lower or in other words vait a ror les, than 15 minutes, the number of customers that are able to be served p inu vill in case. When the service rate is higher the utilization will be lower, $y$ ch makes obability of the customers going away decreases.

\section{Benefits}

- This research can help a restaurant to increase the QOS (Quality of Service), by anticipating if there are many customers in the queue.

- The result of this work may become the reference to to current system and improve the next system. Because the restaurant ca the queue and the number of customers that $111 \mathrm{~g}$.m. each day.

- By anticipating the huge number of custome coming and going in a day, the restaurant can set a target profit that should be chic d daily.

- The formulas that were use ring the completion of the research is applicable for future research and also could be to to erop complex theories.

- The formulas provide echanism model the restaurant queue that is simpler than the Creation of simulatic mo in $[9,4]$

This research pa $b_{a}$ discl sed the application of queuing theory of a Restaurant.

Here we bocu fon the particularly common decision variables. From the result we have obtaine tha he rate which customers arrive in the queuing system is 2.22 customers per minu $\mathrm{nd}$ he service rate is 2.24 customers per minute. The probability of buffer flow if there are or mor customers in the queue is 15 out of 100 potential customers. The probability of buffer o $\mathrm{flow}$ is the probability that customers will run away, because may be they are impatient to y ait in the queue. This theory is also applicable for the restaurant if they want to calculate all the data daily. It can be concluded that the arrival rate will be lesser and the service rate will be greater if it is on weekdays since the average number of customers is less as compared to those on weekends. The constraints that were faced for the completion of this research were the inaccuracy of result since some of the data that we use was just based on assumption or approximation. We hope that this research can contribute to the betterment of a restaurant in terms of its way of dealing with customers.

As our future works, we will develop a simulation model for the restaurant. By developing a simulation model we will be able to confirm the results of the analytical model that we develop in this paper. In addition, a simulation model allows us to add more complexity so that the model can mirror the actual operation of the restaurant more closely [1]. 


\section{References}

[1] T. Altiok and B. Melamed, Simulation Modeling and Analysis with ARENA. ISBN 0 12370523-1. Academic Press, 2007.

[2] D.M. Brann and B.C. Kulick, "Simulation of restaurant operations using the Restaurant Modeling Studio," Proceedings of the 2002 Winter Simulation Conference, IEEE Press, Dec. 2002, pp. 1448-1453.

[3] S. A. Curin, J. S. Vosko, E. W. Chan, and O. Tsimhoni, "Reducing Service Time at a Busy Fast Food Restaurant on Campus," Proceedings of the 2005 Winter Simulation Conference, IEEE Press, Dec. 2005.

[4] K. Farahmand and A. F. G. Martinez, "Simulation and Animation of the Oper woir Fast Food Restaurant," Proceedings of the 1996 Winter Simulation Conference, EEE Press, lec. 1996, pp. 1264- 1271.

[5] K. Kharwat, "Computer Simulation: an Important Tool in T/ F Fast-Foo "nd Astry," Proceedings of the 1991 Winter Simulation Conference, IEEE Pres Pec. 1 a p p. 1-815.

[6] M.Laguna and J. Marklund, Business Process Modeling, Simy amon a Desig. ISBN 0-13091519-X. Pearson Prentice Hall, 2005.

[7] J. D. C. Little, "A Proof for the Queuing Formula: L- N," rations Research,vol. 9(3), 1961, pp. 383-387, doi: 10.2307/167570.

[8] K. Rust, "Using Little's Law to Estimate Cycle Cime and Cost," Proceedings of the 2008 Winter Simulation Conference, IEEE Press, Dec. 08, doi: 10 1109/WSC.2008. 4736323.

[9] T. C. Whyte and D. W. Starks, "ACE: A Pecision To cstaurant Managers," Proceedings of the 1996 Winter Simulation Conferenc Dress, Dec. 1996, pp.1257-1263.

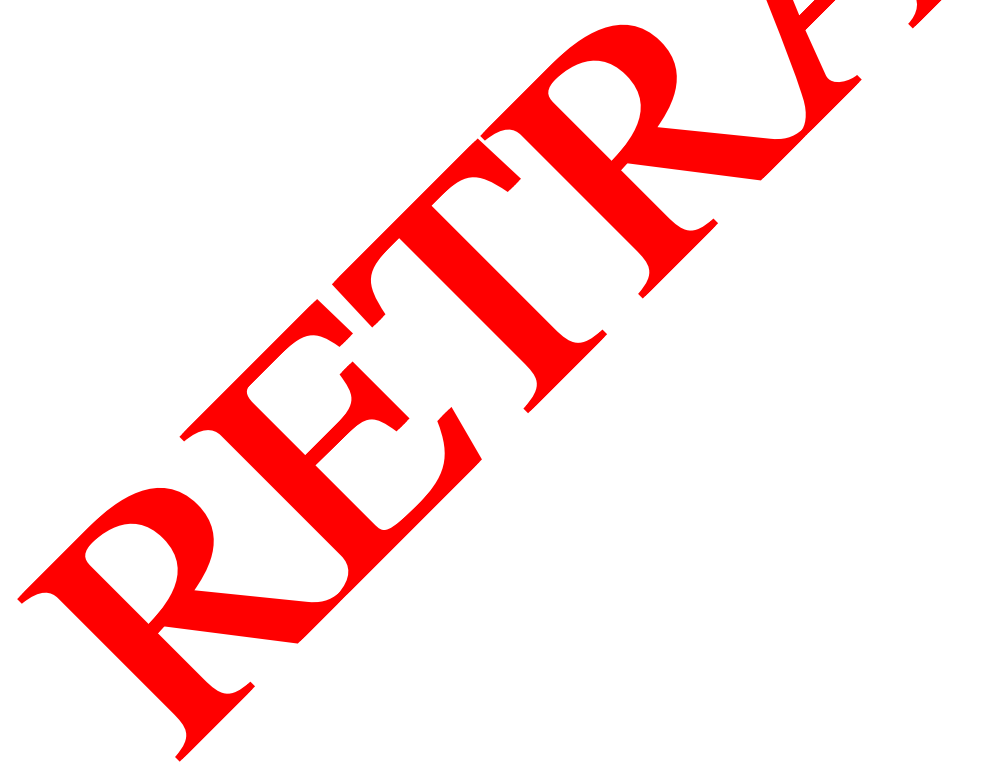

